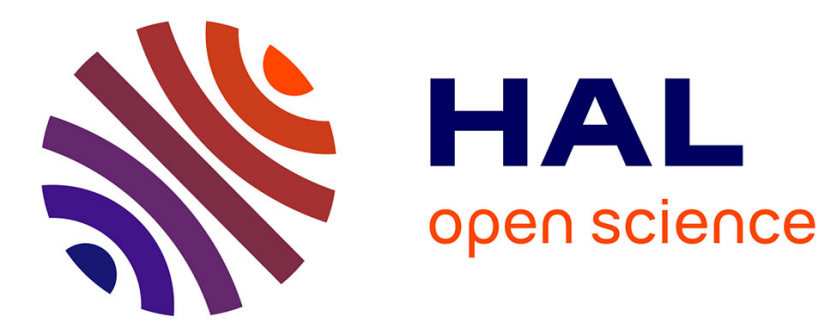

\title{
Le problème du Paléolithique final de Haute-Normandie Bruno Bosselin
}

\section{To cite this version:}

Bruno Bosselin. Le problème du Paléolithique final de Haute-Normandie. Bulletin de la Société préhistorique française, 1983, 80 (10-12), pp.329-334. hal-00201595

\section{HAL Id: hal-00201595 \\ https://hal.science/hal-00201595}

Submitted on 14 Jan 2008

HAL is a multi-disciplinary open access archive for the deposit and dissemination of scientific research documents, whether they are published or not. The documents may come from teaching and research institutions in France or abroad, or from public or private research centers.
L'archive ouverte pluridisciplinaire HAL, est destinée au dépôt et à la diffusion de documents scientifiques de niveau recherche, publiés ou non, émanant des établissements d'enseignement et de recherche français ou étrangers, des laboratoires publics ou privés. 


\title{
Le problème du Paléolithique final de Haute-Normandie
}

\author{
par Bruno A. Bosselin *
}

\begin{abstract}
Résumé : Les recherches récentes ont apporté des éléments nouveaux concernant le Paléolithique final de Haute-Normandie. Des études typologiques prouvent l'existence, comme partout dans le bassin de Paris, de deux industries contemporaines. Le premier groupe se rattache au Magdalénien classique. Par l'importance des troncatures, des becs et des pointes à dos diverses, le second faciès peut être comparé à des ensembles du Tardiglaciaire du Nord-Ouest de l'Europe, (Creswellien et Tjongérien).

Une datation à l'interstade d'Alleröd parait probable.
\end{abstract}

Abstract : Recent investigations have provided new facts about the Upper Palaeolithic of the Normandie. Typological studies appear to indicate the existence, like everywhere in the Paris-basin, of two contemporary industries. The first group can be attributed to the Magdalenian of Périgord. By the development of truncations, borers and arched-backed, angular-backed and shouldered points, the second is compared to assemblages of the Late Upper Palaeolithic of the north-west Europe, (Creswellian and Tjongerian tribes).

Man's occupation is probably contemporaneous with the Alleröd climatic phase.

\section{1 - HISTORIQUE DES RECHERCHES}

S'il fallait définir une chronologie des recherches préhistoriques de Haute-Normandie, il faudrait la diviser en trois périodes fondamentales :

- Jusqu'à la seconde guerre mondiale, le début des recherches.

— La période classique, de 1945 à 1970, dominée par les travaux de F. Bordes et la découverte de la grotte de Gouy.

- L'époque récente, où se sont développées les fouilles d'habitats de plein-air, riches en documents palethnographiques.

Les années 1900 sont marquées par des recherches très fructueuses dans les briquetteries. Les premières séries sont mises au jour et les premiers inventaires publiés, (Coutil, 1894 et Dubus, 1914). Cependant, les découvertes relatives au Paléolithique supérieur restent rares et sans connexion stratigraphique. Seul, l'abri du Mammouth, près de Vernon, a libré un petit

* Résidence du Soleil, 2, chemin de Vénus, Saint-Pierre-du-Bosguérard, 27370 Amfreville-la-Campagne. ensemble magdalénien, (A.-G. Poulain 1902-1912). Un réexamen du matériel a fourni six outils, dont trois grattoirs et un zinken, des lames et lamelles ainsi qu'une faune attribuable au renne (1).

Depuis lors, de nouveaux outillages ont partiellement permis de combler cette absence. En 1950, Bordes et Fitte découvrent, à Evreux, un épipaléolithique sans microlithes, (Bordes et Fitte, 1951). Plus tard, une analogie avec le Magdalénien final a été suggérée, (Bordes, 1974). Le gisement de St-Pierreles-Elbeuf livre sporadiquement quelques pièces dont la pointe " proto-solutréenne », (Bordes, 1954 et Otte, 1974). Avec Goderville et la grotte de Gouy, de nouveaux problèmes sont apparus : ( $F$. Bordes 1963).

- L'existence d'un Paléolithique supérieur initial ; Périgordien 0

- La véritable place chronologique des industries tardives.

(1) Renseignement oral de G. Fosse 
La reprise des coupes de Goderville par différents chercheurs a permis de préciser la position des occupations humaines. Le Périgordien 0 se situe, en fait, au sommet d'un limon du pléniglaciaire inférieur (2). Par contre, la seconde interrogation reste toujours en suspens. Si les sites récemment fouillés ont livré des industries homogènes, les hypothèses sont encore à formuler. A la lumière des dix dernières années, un élément supplémentaire est survenu : le Paléolithique final semblerait se scinder en deux groupes typologiques : le premier, uniquement représenté par St-Pierre-d'Autils, s'apparente au Magdalénien classique tandis que le second révèle certaines influences nordiques et se définit comme suit, (Fosse, 1978) (3) :

- En proportion semblable, les grattoirs sont fréquemment courts (sur éclat ou lame cassée) alors que les burins se retrouvent principalement sur cassure et sur pan naturel.

- Les perçoirs atteignent environ $5 \%$ de l'outillage.

- Les lames tronquées sont nombreuses et typiques.

- Le groupe typologique le plus important est celui des pièces à dos. A côté des pointes à dos courbe diverses du Magdalénien français (pointe azilienne - lamelle à dos), on trouve quelques dos associés à une troncature, des dos anguleux et parfois même à cran (pointe de Hambourg et de Petersfels).

La répartition des différents outils et la présence d'éléments insolites permettent une attribution à un stade avancé du Paléolithique. La tradition nordique, sous-jacente, est encore à cerner afin d'établir des filiations plus précises. En effet, le phénomène présente des similitudes avec l'occupation du Bassin Parisien à la même époque. De plus, les crans, existant dans le Magdalénien VI du Périgord, n'atteignent jamais un pourcentage si élevé. Il est donc difficile d'imaginer une brutale apparition de ces outils sans apport extérieur. De ce fait, deux théories paraissent plausibles ;

- Un Epimagdalénien d'un faciès proche des industries à Federmesser et dérivant d'un Magdalénien local.

- Un faciès septentrional du Magdalénien, contemporain du phylum classique et provenant des cultures nordiques immédiatement antérieures.

Pour étayer ces données, nous allons comparer les industries, (Laplace, 1964). Les analogies entre habitats, plus restreintes ne concerneront que StPierre-du-Bosguérard, (Bosselin, 1982).

\section{2-COMPARAISONS TYPOLOGIQUES}

\section{Avec le Nord-Ouest de l'Europe}

Le caractère encore franchement épipaléolithique de nos outillages élimine à priori toutes les cultures

(2) Après une fouille à plat de G. Fosse et J.-P. Lautridou.

(3) Il comprend les gisements d'Ambenay, (G. Fosse), d'Evreux, (Bordes et Fitte, 1951), de St-Pierre-du-Bosguérard, (Bosselin, 1982) et de Vattetôt s/s Beaumont, (Verron, 1977-1979). contemporaines ou postérieures au Dryas III. On peut donc, d'ores et déjà, exclure l'Ahrensbourgien. A un moindre degré, l'absence de zinken et la mauvaise facture des pointes à dos et troncature empèchent tout rapprochement strict avec le Hambourgien.

En ce qui concerne le Creswellien, il a été défini par quelques sites de Grande-Bretagne, (Campbell,
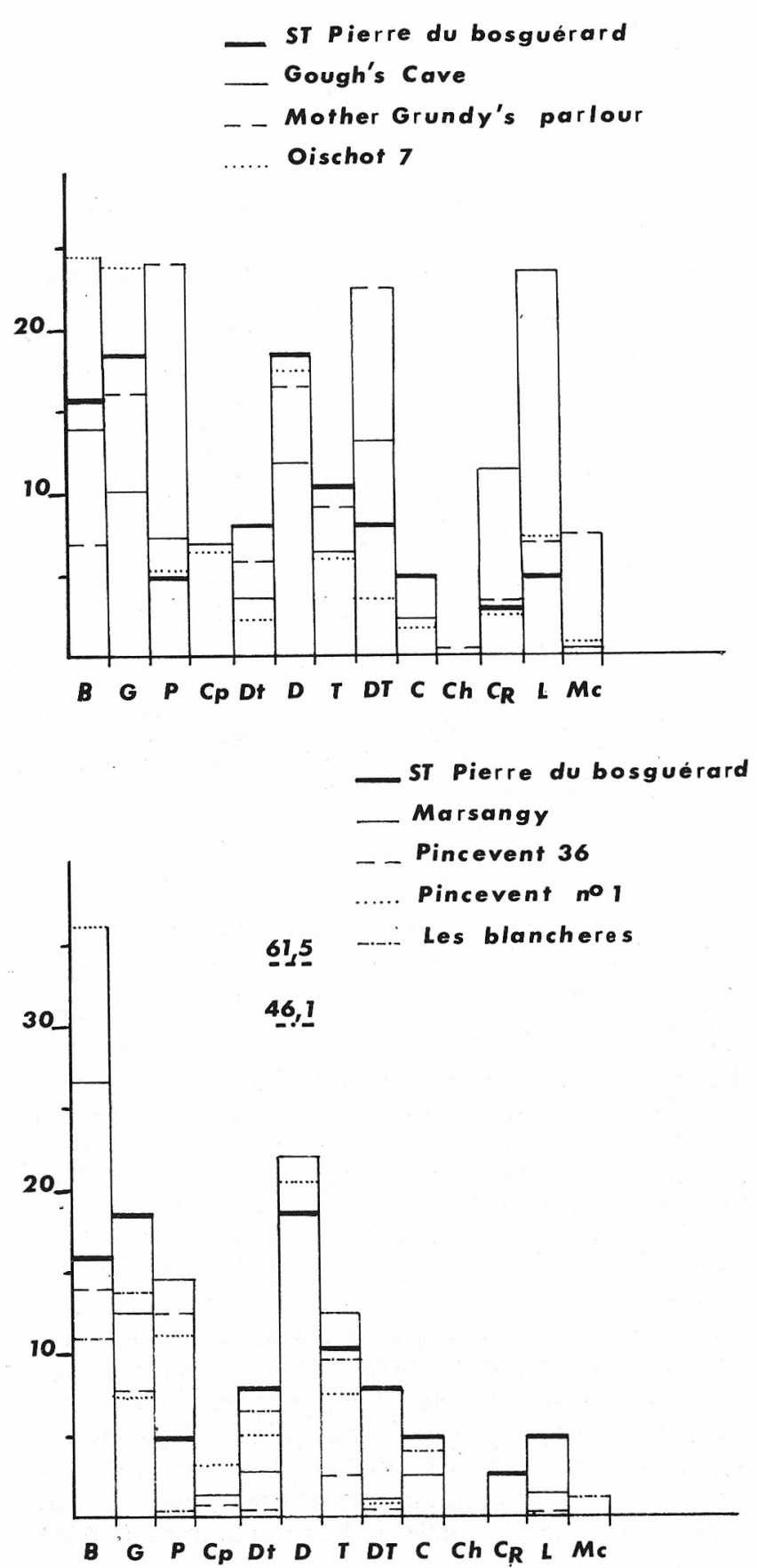

Fig. 1 - Comparaisons typologiques avec le Bassin Parisien et le Nord-Ouest de l'Europe. 
1980) : la série de Gough's Cave étant la plus représentative. On note en général une légère prédominance des burins, dièdres et sur pan naturel, sur les grattoirs, longs. Les zinken sont présents tandis que, parmi les pièces à dos, on signale l'existence de pointes de Creswell. Dans l'ensemble, (fig. 1), les profils typologiques sont semblables malgré quelques dissemblances :

- Absence de grattoirs courts,

- Prédominance minime des burins,

- Foisonnement des lames retouchées.

La comparaison avec la Haute-Normandie amène certaines remarques: Le Creswellien moyen, (Gough's Cave) parait trop peu évolué alors que le stade final, (Mother Grundy's Parlour) est à la limite de l'Epipaléolithique. Cela nous montre que, malgré les points communs, les deux industries ne sont pas totalement identiques. Les gisements normands se situeraient plutôt dans une période intermédiaire entre un Creswellien moyen et final, soit vers 14000-11000 B.P.

Le Tjongérien a été profondément étudié en Belgique et aux Pays-Bas, notamment par A. Bohmers et F.L.Van Noten, (Bohmers, 1961 et Van Noten, 1967) ainsi qu'en Allemagne par H. Schwabedissen, (Schwabedissen, 1954). Il possède les caractères suivants :

- Les grattoirs, surtout sur éclat, dépassent légèrement les burins d'angle et sur troncature.

- Les perçoirs sont rares et les zinken absents.

- Les lames tronquées atteignent parfois $10 \%$.

- Les pièces à dos se rencontrent sous plusieurs formes: pointes à dos courbe, Tjonger, pointe à dos anguleux et à cran.

L'évolution montre une raréfaction des burins et des lamelles à dos, un passage progressif des dos anglés vers les crans et un développement massif des microlithes.

De nouveau, cette industrie s'accorde en partie avec les notres, mis à part, peut-être, les supports des armatures et les burins sur troncature en défaut dans les sites normands. Une attribution à un stade avancé, mais pas encore final, parait satisfaisante. Les quelques disparités qui empèchent un rapport étroit entre les deux régions peuvent s'expliquer de différentes manières :

- La distance entre l'Allemagne d'une part et la Grande-Bretagne d'autre part et la Haute-Normandie est assez élevée. Cela introduit nécessairement un facteur géographique non négligeable dont il faudra tenir compte dans les conclusions.

- La pauvreté des séries normandes tient un role dans le phénomène statistique. Ainsi, la forte proportion de certaines pièces, si elle traduit bien une tradition lithique, peut aussi être amplifiée par cette faiblesse.
- L'hypothèse des migrations ne doit pas être écartée. En effet, le nomadisme et surtout la formidable explosion démographique contemporaine du Magdalénien ont certainement poussé l'homme vers de nouvelles conquêtes. La répartition des habitats de cette période montre des zones fortement peuplées, véritables foyers de culture, au milieu de déserts, (Rozoy, 1978, p. 1107). De ce fait, l'exode d'une population septentrionale, lors d'un refroidissement climatique de la fin du Würm, aurait très bien pu créer un groupe, dérivé de la culture originelle et ayant évolué tout en conservant ses traditions. Cela justifierait les analogies précédentes.

\section{Avec le Bassin Parisien}

Dans l'ensemble, le Magdalénien et l'Azilien du Bassin Parisien présentent une homogénéitế certaine. Cependant, les travaux de A. Leroi-Gourhan à Pincevent ont montré l'existence de deux faciès parallèles (fig. $\left.n^{\circ} 1\right)$ :

- Le premier se caractérise par la faiblesse des lamelles à dos, l'importance numérique des lames tronquées et des becs. Cela correspond à l'habitation $n^{\circ} 1$, (Leroi-Gourhan, 1966) et ne semble pas à l'abri d'influences nordiques, surtout creswelliennes, (Schmider, 1982).

- Le second, représenté par la section 36, (LeroiGourhan, 1972) se rapproche du Magdalénien classique avec de nombreuses lamelles à dos, des burins bec-de-flute et des micro-perçoirs.

Des études récentes ont démontré des filiations entre ces deux groupes et les cultures épipaléolithiques. Le premier donnerait un Epipaléolithique du type des Blanchères, (Schmider, 1971) ; le gisement de Marsangy dans l'Yonne (4) assurant la continuité pour le stade paléolithique final, (Schmider, 1979). Par contre, le second aboutirait par l'intermédiaire de l'Azillien aux faciès mésolithiques.

On peut évidemment effectuer une analogie entre ces deux régions. On doit donc y reconnaitre une occupation similaire bien que les industries soient quelques peu distinctes. Sur ce modèle, on peut esquisser un tableau de répartition pour la HauteNormandie, (fig. 2).

\section{3-COMPARAISONS ETHNOGRAPHIQUES}

De telle comparaisons ne sont possibles qu'avec l'habitat de St-Pierre-de-Bosguérard, (Bosselin, 1982). Du fait de l'éloignement, nous avons volontairement limité notre échantillon d'étude à deux sites

(4) B. Schmider assimile l'industrie du Pré des Forges à Marsangy au groupe de Thayngen en Suisse. 


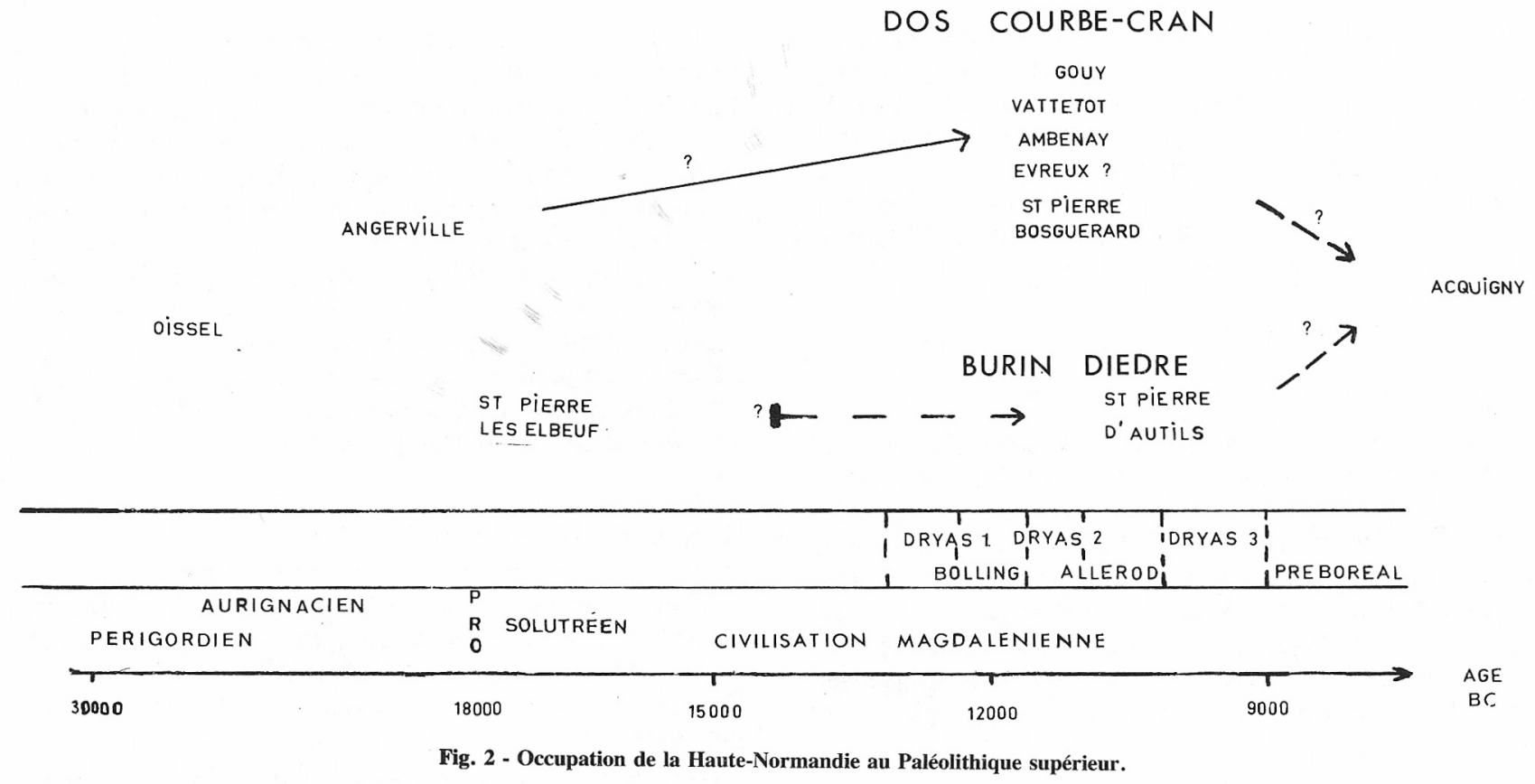

très voisins, proches d'Ahrensburg (5) ; Borneck et le Pinnberg. Ils s'étagent depuis le Dryas I, (Borneck-Hambourgien) jusqu'au Dryas III, (Le Pinnberg) en passant par Alleröd, (Borneck-Magdalénien). Ces travaux proviennent essentiellement de fouilles de A. Rust republiées à l'occasion d'un mémoire de maitrise, (A. Rust, 1951 et 1958 a et b et G. Fosse, 1971).

Borneck-Hambourgien (fig. 3, $n^{\circ} 2$ )

L'habitat de Borneck, fouillé en 1946, se compose de deux cercles concentriques. La répartition au sol montre les particularités suivantes :

- $\mathrm{Au}$ nord, les pierres étaient plus grosses et plus espacées.

- Près de l'entrée, se trouvaient deux foyers ainsi que des groupements de pierres qui constituaient, selon $\mathrm{A}$. Rust, des sièges.

- Les silex taillés permettent quelques remarques ethnographiques. La transformation de la matière première s'effectuait à l'extérieur. La rareté des vestiges à l'intérieur implique la délimitation d'une zone de repos et de sommeil : le second foyer confirmant les dires de A. Rust. L'espace libre entre les deux tentes, très éclairé, aurait pu servir pour des taches techniques: l'absence de matériel osseux empéchant toute affirmation.

(5) Les structures d'habitat semblent, à notre connaissance, absentes de Grande-Bretagne.
D'après l'auteur, il s'agirait d'un habitat saisonnier. Du fait de l'attribution au Dryas I, cela n'était possible que l'été.

Les silex sont, comme à St-Pierre (fig. 3, $\mathrm{n}^{\circ} 1$ ), principalement à l'extérieur. On retrouve l'amas de débitage près de l'entrée ainsi que des pierres de calage. La seule originalité est l'existence à Borneck d'une délimitation interne. Tout se passe comme si les préhistoriques avaient consacré une zone pour le sommeil et un espace destiné au travail. Il ne semble pas que cela soit le cas en Haute-Normandie.

Le Pinnberg (fig. 3, $n^{\circ}$ 6)

Le site a livré les restes de six huttes qui s'étalent du Dryas III au Mésolithique moyen. Les structures, assez simples, consistent en des fosses remplies de cendres et des trous de poteau. Il n'y a pas de foyer. $\mathrm{Du}$ fait de l'ancienneté des fouilles, il n'existe pas dans les publications de plans de répartition.

Il est regrettable que les renseignements soient si peu nombreux car nous avons là une forme et une superficie extrèmement proche de la notre, $(3 \mathrm{~m} \times$ $2,5 \mathrm{~m}$ ).

Le décalage chronologique renforce encore plus l'idée d'une migration, venue du Nord lors d'une période froide pour s'installer chez nous: une évolution légèrement différente ayant forgé ces originalités. Par conséquent, nous allons pouvoir préciser les facteurs responsables de la singularité de nos industries tardives. 

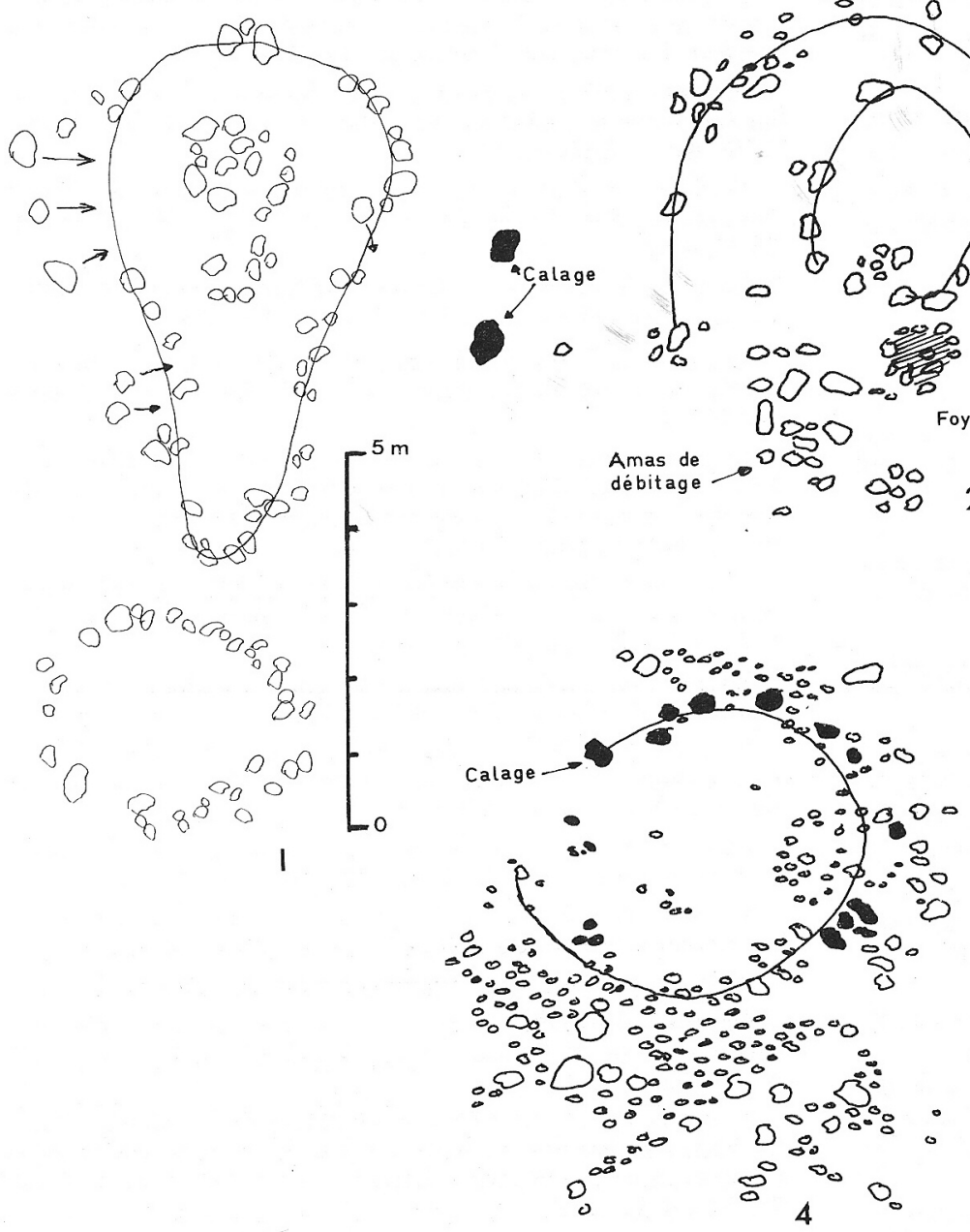

$D$<smiles>[18OH]</smiles>

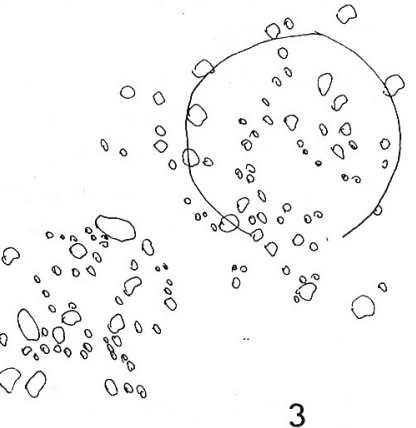

Amas de :̊: débitage
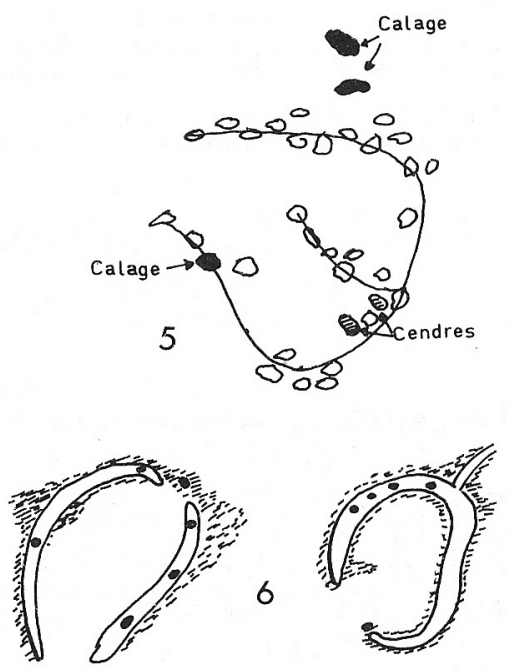

Fig. 3 - Comparaisons ethnographiques avec le Nord-Ouest de l'Europe.

\section{4-CONCLUSIONS}

Les recherches préhistoriques de ces dix dernières années ont permis de scinder le Paléolithique final en Haute-Normandie en deux groupes. Le premier, porteur d'une tradition magdalénienne semble très rare. Le second, d'essence septentrionale et très nettement majoritaire, se rapproche d'un Tjongérien évolué et, à un moindre degré, d'une culture creswellienne. Cette occupation bipolaire se retrouve partout dans les environs : elle est en effet reconnue pour le Bassin Parisien ainsi que pour le Bassin de la Somme, (Fagnard, 1980).

Le manque de précisions ressortant des comparaisons ethnographiques nous montre que la datation de chaque site peut s'étendre depuis le Dryas I jusqu'au Dryas III . De ce fait, l'hypothèse la plus plausible est la suivante. D'une part, des préhistoriques ont perpétué la tradition classique du Périgord ; le stade final étant représenté par St-Pierre-d'Autils. D'autre part, on peut penser que, lors d'une période froide, (Dryas I ou II), des habitants venus du nord, (Hambourgien d'Allemagne ou Maisiérien ou Creswellien ancien de Grande-Bretagne) se seraient installés dans nos régions. Par la suite, ils auraient évolué de manière autochtone en Normandie. Nous aurions alors un groupe au même titre que ceux du Bassin Parisien et d'Europe du Nord. Cela nous semble, malgré les réserves quant aux séries normandes très limitées, une hypothèse de travail méritant d'être développée. Les relations avec les gisements d'Ile-de-France seraient alors plus simples. Une évolution différente, des contacts humains moins fréquents avec l'autre groupe auraient forgé l'originalité de ces traditions.

En conclusion, on peut dire que nous avons, à coté d'un Magdalénien indéterminé, une industrie datant de l'extrême fin du Paléolithique, sans doute Al- 
leröd, qui n'est rattachable à ancune civilisation connue mais qui ressemble au Tjongérien et au Creswellien.

Nous proposons donc le terme de Paléolithique final de tradition nordique pour ces séries. La découverte dans l'avenir de gisements similaires, mais plus riches, permettra, on l'espère, de préciser les orientations formulées présentement. (6)

Bohmers A. (1961) - Statistiques et graphiques dans l'étude des industries lithiques préhistoriques ; considérations générales sur le Hambourgien, Tjongérien Magdalénien et Azilien. Paléohistoria, vol. 8, 1961, pp. 15-37, 7 fig., 1 pl.

Bordes F. (1954) - Les limons quaternaires du bassin de la Seine : stratigraphie et industrie préhistorique. Archives de l'I.P.H., Mémoire 26, 1954, 473 p., 175 fig., 34 tabl.

Bordes F. (1963) - Les loess de Goderville et la stratigraphie du quaternaire récent. Soc. Géol. Fr., 7e série, t. 5, 1963, pp. 443-445.

Bordes F. et Fitre P. (1951) - Une industrie épipaléolithique à Evreux. Bull. Soc. Préhist. Fr., t. 48, 1951, pp. 147-154, 3 fig.

Bordes F. et alt. (1974) - L'industrie de la grotte ornée dé Gouy (Sne-Mme). Bull. Soc. Préhist. Fr., t. 71, n 4, 1974, pp. 115-118, 2 fig.

Bosselin B. (1982) — L'habitat épimagdalénien des Bruyères ; essai d'analyse et d'interprétation des structures. Bull. Soc. Préhist. Fr., t. 79, n 5, 1982, pp. 138-147, 7 fig.

Campbell J. (1971) - The Upper Paleolithic of Britain. St John's college, Oxford, 1971, 447 p., 106 fig.

Campbell J. (1980) - Le problème de la subdivision du Paléolithique supérieur britannique dans son cadre européen. Soc. Royale Belge d'Anth. et de Préhist., 1980, vol. 91, pp. 19-77, figures.

CoutiL L. (1893) - Résumé des recherches préhistoriques en Normandie. Soc. Norm. Et Préhist. et Hist., t. 1, 1893, pp. 34-140, fig.

Dubus A. (1914) - Carte pré et protohistorique du département de la Seine-Inférieure. ibid, t. 22, 1914-1915, pp. 1-111.

(6) Je tiens à remercier toutes les personnes qui m'ont aidé pour cette recherche. En premier lieu, J.-P Watté pour ses conseils et ses encouragements, ceux qui m'ont prété des documents nécessaires R. Bayon et G. Fosse ainsi que les chercheurs qui, par leur correspondance, m'ont guidé : $\mathrm{J}$. Gaussen, J.-G. Rozoy, B. Schmider et A. Thevenin.
Fagnard J.-P. (1980) - Le Paléolithique supérieur dans le bassin de la Somme d'après les recherches de V. Commont. Soc. Archéo. Picardie, vol. 7, 1980, pp. 19-32, 12 fig.

Fosse G. (1971) - Les structures d'habitat en Europe du nord au Paléolithique final et au Mésolithique. Maitrise, Faculté Paris I, UER Art et Archéo., 1971.

Fosse G. (1978) - Le Paléolithique supérieur de HauteNormandie. Soc. Norm. Et. Préhist. et Hist., t. 44-4, 1978, pp. 23-33, 4 fig.

LAPLACE G. (1964) - Essai de typologie systématique. Annali del Universita di Ferrarra, 1964, Sezione XV, $2^{\mathrm{C}}$ supp.

Leroi-Gourhan A. et Brezillon M. (1966) - L'habitation $n^{\circ} 1$ de Pincevent près de Montereau. Gallia Préhist., t. 9, nº 2, 1966 , pp. 263-385.

Leroi-Gourhan A. et Brezillon M. (1972) - Fouilles de Pincevent. Essai d'analyse ethnographique d'un habitat magdalénien : la section $36.7^{\text {e }}$ Supp. à Gallia Préhist., 1972, 331 p., 199 fig., 4 annexes, plan hors-texte.

Отте M. (1974) - Une hypothèse d'interprétation de la pointe proto-solutréenne de St-Pierre-les-Elbeuf. Bull. Soc. Préhist. Fr., t. 71, no 7, 1974, pp. 196-198, 1 fig.

Poulain A.-G. - Les fouilles de l'abri du Mammouth. Soc. Norm. Et. Préhist. et Hist., 1902 à 1911.

Rozoy J.-G. (1978) - Les derniers chasseurs. Soc. Archéo. Champenoise, Juin 1978, 3 tomes, 1256 p., 294 fig., 1 tome de 259 fig., planches et tableaux hors-texte.

Rust A. (1951) - Préhistoire du Nord-Ouest de l'Europe. L'Anthropologie, t. 55, 1951, pp. 205-218, 6 fig., 1 tabl.

Rust A. (1958) - Die Jungpaläolithischen Zeltanlagen von Ahrensburg. Neumünster, Offa Bücher, 1958, nº 14.

Rust A. (1958) - Die Funde vom Pinnberg. ibid, no 15.

SCHMider B. (1971) - Les industries lithiques du Paléolithique supérieur en Ile-de-France. $6^{\mathrm{C}}$ Supp. à Gallia Préhistoire, 1971, 218 p., 109 fig.

SCHMIDER B. (1979) - Un nouveau faciès du Magdalénien final du bassin parisien : l'industrie du pré des Forges à Marsangy. (Yonne) in la fin des temps glaciaires, 1979, C.N.R.S., t. 2, pp. 763-771, 4 fig., tabl.

Schmider B. (1980) - The Magdalenian culture of the Paris-river bassin and its relationship with the Nordic cultures of the late old stone age. World Archaeology, vol. 14, 1982, $\mathrm{n}^{\circ} 2$, pp. 259-269, 4 fig., tabl.

Schwabedissen H. (1954) - Die Federmesser Gruppen des nordwesteuropäischen Flachlandes. Neumünster, 1954, 104 p., 23 fig., 106 planches.

VAn Noten F.-L. (1967) - Le Tjongérien en Belgique. Soc. Royale Belge d'Anthr. et de Préhist., t. 78, 1967, pp. 197-236, 5 fig.

Verron G. (1977) - Informations archéologiques. Gallia Préhistoire, 1977-1979, t. 20-2 et 22-2. 